\title{
Highly Suspected COVID-19 Cluster with Multiple Negative SARS-CoV-2 RNA Tests: A Case Report
}

\author{
Xialing Huang $\mathbb{D}^{\prime}$ \\ Xiaoping Long $\left(\mathbb{D}^{2}\right.$ \\ Jun $\mathrm{Zi}^{3}$ \\ Ping $\mathrm{Liu}^{4}$ \\ 'Department of Infectious Diseases, \\ Leiyang People's Hospital, Leiyang, \\ Hunan, 42I800, People's Republic of \\ China; ${ }^{2}$ Pulmonary and Critical Care \\ Medicine, The First Affiliated Hospital of \\ University of South China, Hengyang, \\ Hunan, 42100I, People's Republic of \\ China; ${ }^{3}$ Department of Pediatrics, \\ Leiyang, Hunan, 421800, People's \\ Republic of China; ${ }^{4}$ Department of \\ Emergency, People's Hospital of Leiyang \\ City, Leiyang, Hunan, 421800, People's \\ Republic of China
}

Correspondence: Xiaoping Long Pulmonary and Critical Care Medicine, The First Affiliated Hospital of University of South China, Hengyang, Hunan, 421001 , People's Republic of China $\mathrm{Tel}+8613975403734$

Email xiaopinglong88@sina.com

\begin{abstract}
The patient had several close contacts with friends from Wuhan, the epicenter of the epidemic. His mother and father had close contact with him. His father was later diagnosed with COVID-19 infection after a positive reverse transcription PCR test for SARS-CoV-2 RNA. The patient and his mother were diagnosed as suspected cases of COVID-19 based on a history of exposure, clinical manifestation, and imaging examination. However, the patient was tested more than three times with the reverse transcription PCR test for SARS-CoV-2 RNA, and the results were negative each time. COVID-19 should be suspected, regardless of SARS-CoV-2 test negativity, for recent close contact with a confirmed case and respiratory symptoms.
\end{abstract}

Keywords: COVID-19, respiratory symptoms, suspected, negative, cluster

\section{Introduction}

Coronavirus disease 2019 (COVID-19), which is caused by severe acute respiratory syndrome coronavirus 2 (SARS-CoV-2), broke out in December 2019. The global epidemic caused by COVID-19 continues. Currently, it is a major global public health event. ${ }^{1}$ Patients with COVID-19 are the main sources of infection. ${ }^{2}$ The disease is infectious in its incubation period. However, the source, virulence, and clinical manifestations of the virus are not fully understood. There are two primary detection methods for SARS-CoV-2. SARS-CoV-2 RNA by real-time reverse transcriptase (RT) PCR or SARS-CoV-2-specific immunoglobulin (Ig) M and IgG antibodies can be detected. However, at the beginning of the outbreak when our knowledge of COVID-19 was limited, false negative SARS-CoV-2 RNA results were a concerning problem. This paper reports the diagnosis and treatment of 2 patients who were highly suspected to have COVID-19. They were close contacts of 1 patient with confirmed COVID-19 but their SARS-CoV-2 RNA results were negative multiple times.

\section{Case Presentation Chief Complaints}

On January 11, 2020, the patient, an 18-year-old male, and his father, a 41-year-old male, drove to the railway station to pick up a friend from Wuhan and then they had dinner together. The patient had dinner two times with friends who had traveled from Wuhan in the subsequent days ( $4-5 \mathrm{~h}$ the first time and $3 \mathrm{~h}$ the second time). On January 15, 2020, 
he was in contact for $2 \mathrm{~h}$ with another friend who traveled back from Wuhan. The patient resided with his father and mother, a 40-year-old female. His mother became ill on January 16, 2020. The patient became ill on January 17, 2020, and his father became ill on January 18, 2020.

The local clinic gave the patient and his parents antiinfection treatment, but their cough did not improve. They were treated with therapy involving "ribavirin, levofloxacin, clindamycin, dexamethasone" (details unknown). The patient and his parents came to Leiyang People's Hospital for treatment. They were admitted to the isolation ward in the Infection Department of the People's Hospital of Leiyang City, Hunan, China on January 23, 2020.

\section{History of Present Illness}

The symptoms of the three patients were similar to the common cold, mainly fever and cough. The patient and his parents did not have chills, chest tightness, shortness of breath, hemoptysis, chest pain, nasal congestion, runny nose, or muscle soreness.

\section{History of Past IIlness}

The patient and his parents had no remarkable past history.

\section{Personal and Family History}

The patient and his mother did not have a significant personal history. The patient's father had hepatitis B.

\section{Physical Examination}

The detected temperature, pulse, respiration, blood pressure, and oxygen saturation of the patient and his parents were within normal range. No obvious abnormalities were seen after examination of the heart, lungs, abdomen, and limbs.

\section{Laboratory Testing}

The patient had an abnormally high erythrocyte sedimentation rate $(31 \mathrm{~mm} / \mathrm{h}$, normal range: $0-20 \mathrm{~mm} / \mathrm{h})$. Routine blood tests were normal for the patient and his parents. Liver function, renal function, myocardial enzymes, electrolytes, coagulation function, procalcitonin concentration, and D-dimer were within normal ranges. The IgM tests of influenza A, influenza B, seven respiratory viruses, and Mycoplasma were negative.

\section{Imaging Examination}

Pulmonary high resolution computed tomography (HRCT) was performed on the patient and his parents. Features of COVID-19 pneumonia, including bilateral multifocal ground glass opacity, peripheral distribution, and multilobe involvement, were identified in the patient (Figure 1A and B), his mother (Figure 2A and B), and his father (Figure 3A and B).

\section{Genetic Testing}

The patient and his parents were tested for SARS-CoV-2 repeatedly during hospitalization. His father tested positive for SARS-CoV-2 by RT-PCR after the second oropharyngeal swab sample. However, the patient and his mother tested negative three times for SARS-CoV-2 RNA by RTPCR of nasopharyngeal swab samples.

\section{Multidisciplinary Expert Consultation}

An expert group composed of doctors from the Respiration Department, Infectious Disease Department, Imaging
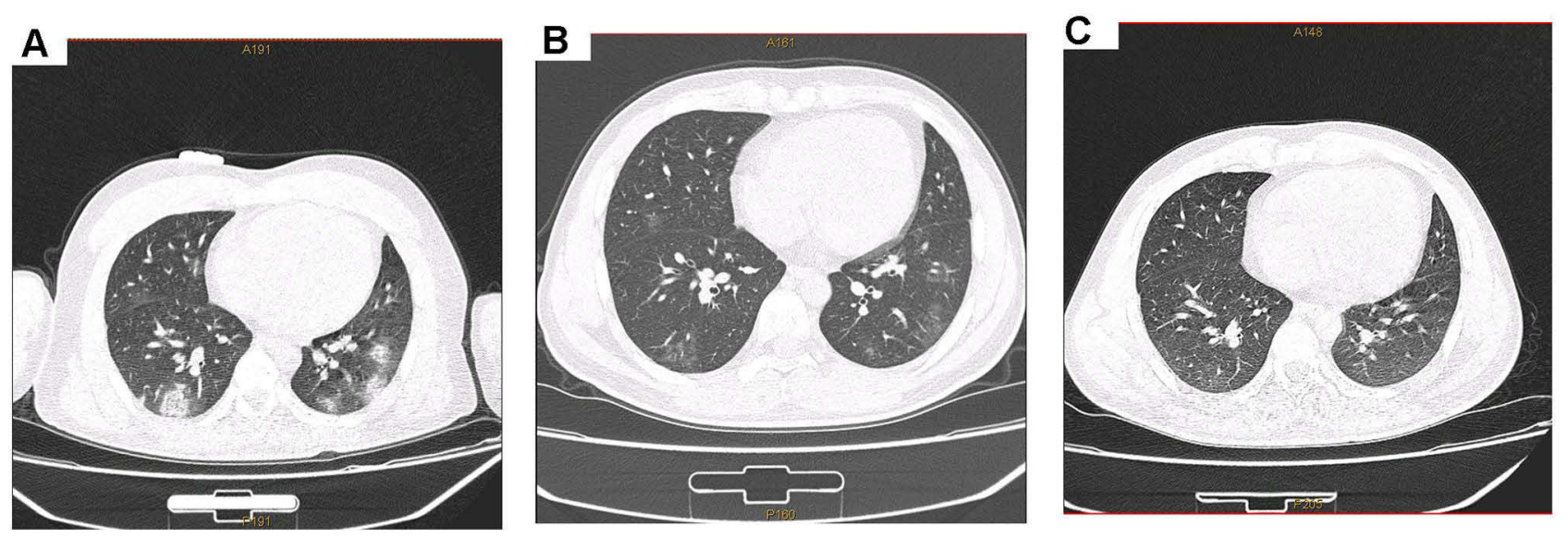

Figure I Chest computed tomography scan results of the 18-year-old male patient's lung. (A) On January 23, 2020, a computed tomography (CT) scan of the patient showed patchy ground glass density shadows distributed in both lungs and bilateral pleura; (B) Chest CT examination on February I0, 2020 showed that the lung lesions were obviously absorbed; (C) CT scan images on February 28, 2020 revealed normal lungs. 

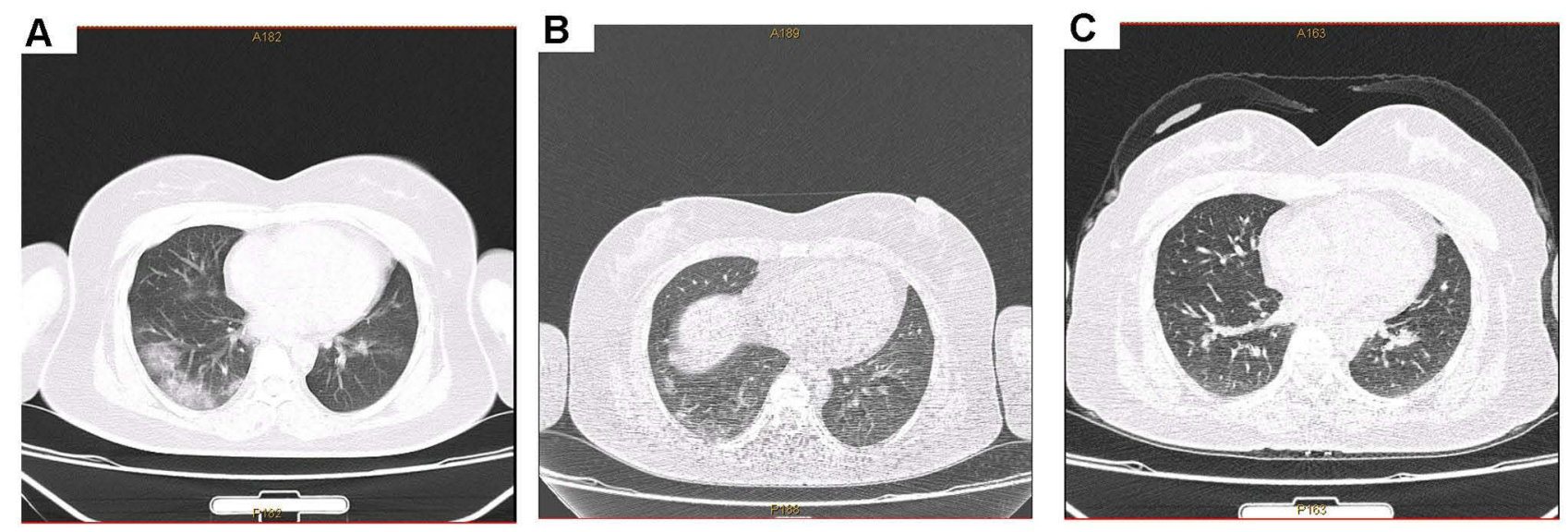

Figure 2 Computed tomography scan results of the patient's mother's lungs. (A) On January 23, 2020, computed tomography (CT) scan of the patient's mother, a 40-yearold female, showed ground glass density shadow under the pleura of both lungs and the bilateral middle and lower lungs; (B) Chest CT examination on February 6, 2020 showed that the lung lesions were obviously absorbed; (C) CT scan images on February 24, 2020 showed that most of the multiple exudative foci in both lungs were absorbed and dissipated.

Department, Hospital Infection Management Branch, etc. was consulted for the cases of the patient and his parents.

\section{Final Diagnosis}

According to the "Diagnosis and Treatment Protocol for Novel Coronavirus Pneumonia" (7th Trial Version) ${ }^{2}$ and after consultation with the COVID-19 expert group, the patient's father was diagnosed with a confirmed case of COVID-19. The patient and his mother were diagnosed with suspected cases of COVID-19.

\section{Treatment}

Relevant clinical treatment was carried out for the patient and his parents, following guidance from the "Diagnosis and Treatment Protocol for Novel Coronavirus Pneumonia" (7th Trial Version). ${ }^{2}$ They were given "levofloxacin tablets $0.5 \mathrm{~g}$ oral Qd" to fight atypical pathogen infection, "injection of recombinant human interferon $\mathrm{a}-1 \mathrm{~b}$ $60 \mathrm{mg}$ inhalation Bid" and "oseltamivir capsule $75 \mathrm{mg}$ oral Bid" antiviral treatment from January 23, 2020. On February 2nd, it was changed to oral antiviral therapy with arbidol tablets.

\section{Outcome and Follow-Up}

About 4 wk after the onset of symptoms, the patient and his parents recovered. The patient and his parents were hospitalized for 15 days and then quarantined to their home for 2 wk post-discharge. None had respiratory symptoms, such as fever and cough. Pulmonary HRCT scan showed that the lung lesions were absorbed in the patient on February 28, 2020 (Figure 1C). His parents had similar resolution of the lung lesions (Figures $2 \mathrm{C}$ and $3 \mathrm{C}$ and D).

While the patient was quarantined for $2 \mathrm{wk}$, he underwent four more SARS-CoV-2 tests. Each came back negative. However, IgM and IgG tests for SARS-CoV-2 were positive on March 4, 2020.

All three cases continued to be observed at home for 2 wk after discharge. Because the son wanted to return to school, he attended a follow-up examination in the third week after discharge. The IgM and IgG tests for SARSCoV-2 were positive on March 4, 2020. He, alone, was returned to the isolation hotel for further observation; during this period, four additional RT-PCR tests for SARSCoV-2 RNA ( $>24-\mathrm{h}$ intervals) gave negative results. After seven rounds of negative SARS-CoV-2 RNA test results, the patient was discharged to home.

\section{Discussion}

This case report introduced 2 patients diagnosed with highly suspected COVID-19 who were in contact with a patient with confirmed COVID-19 and tested negative at least three times for SARS-CoV-2 RNA. This family cluster was in close contact with friends who traveled to Wuhan and a resident of Wuhan, where the first cases of COVID-19 were reported. They shared obvious respiratory symptoms like fever, cough, sputum expectoration, and labored breathing. Guan et $\mathrm{al}^{3}$ found that fever and cough were the most common COVID-19 symptoms (with $88.7 \%$ and $67.8 \%$ of the patients, respectively, exhibiting during hospitalization). This study also found that routine blood tests were normal, inflammatory indexes were not 

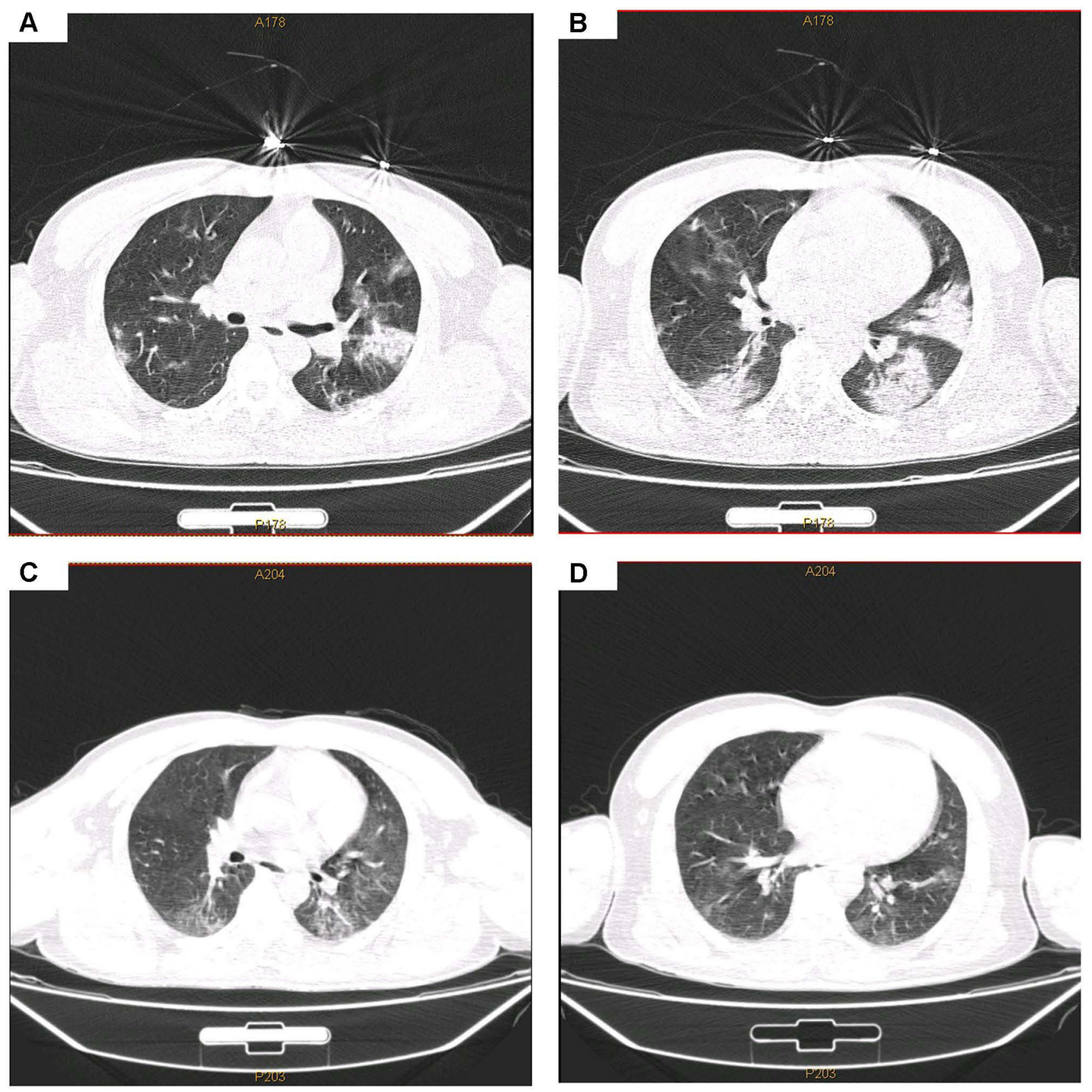

Figure 3 Computed tomography scan results of the patient's father's lung. (A, B) On January 25, 2020, multiple exudates and consolidation foci were found in both lungs of the patient's father, $4 \mathrm{I}$-year-old male. Ground glass appearance with distribution under the chest was observed; (C, D) Computed tomography scan images on February $2 \mathrm{I}$, 2020 revealed an improvement in the abnormalities.

high, and obvious pulmonary medical imaging changes occurred within 1 wk after symptom onset after analyzing the data of 1099 COVID-19 patients.

A previous study identified typical pulmonary HRCT imaging features of COVID-19 pneumonia, including bilateral multifocal ground glass opacity, peripheral distribution, multi lobe involvement, and lower lobe elevation. ${ }^{4}$ The imaging findings of the patient and his parents were consistent with the features above. We observed that the patient's father had more serious clinical symptoms and imaging changes, and the complete recovery time of the lung lesions was longer than the patient and his mother. Elderly male patients may be more susceptible to SARS-CoV-2 infection.

Although there are no clinically approved antiviral drugs for COVID-19, clinical trials of several known antiviral drugs, their combination as well as development of vaccines are ongoing. Antiviral drugs, vaccines, antiSARS-CoV-2 antibody treatments, and convalescent 
plasma therapy are currently under extensive research and clinical trials for the treatment of COVID-19. ${ }^{5}$ Despite the lack of proven effective antiviral drugs, our treatment plan was effective for this family. Because the patient and his parents had no underlying diseases, oral antiviral therapy, traditional Chinese medicine, and other symptomatic support treatments were administered. Their lung imaging improved significantly, and the patient and his parents recovered completely.

Clinicians have observed that some COVID-19 positive cases confirmed by pulmonary HRCT have negative RT-PCR results. A study of 610 patients with COVID-19 found a high false-negative rate of RT-PCR results for SARS-CoV-2. ${ }^{6}$ Viral load in the human body was an important factor related to the severity and disease course that affected the detection, and the viral load was varied among samples. In another study, SARS-CoV-2 RNA detection varied depending on where the sample was collected. In the detection of SARS-CoV-2 RNA of 132 confirmed COVID-19 cases, the positive rate from nasopharyngeal swabs was $38.13 \%(180 / 472)$, and the positive rate from sputum was $48.68 \%$ (148/304 times). The viral load of sputum samples was higher than that of throat swabs. ${ }^{7}$ One study reported that SARS-CoV-2 RNA can be detected in bronchoalveolar lavage fluid samples of critically ill patients but not in the upper respiratory tract samples of some patients. ${ }^{8}$ The detection of SARS-CoV-2 RNA in bronchoalveolar lavage fluid samples as a supplementary intervention measure has a certain reference value and clinical significance for guiding the prognosis of the disease. ${ }^{9}$ It was also reported that some patients recovering from suspected COVID-19 will eventually test positive for SARS-CoV -2 RNA. ${ }^{10}$

This report emphasizes that the comprehensive consideration of exposure history, clinical manifestations, laboratory tests, and imaging findings play an important role in making an initial COVID-19 diagnosis and guiding early isolation. In one report, the final diagnosis of a patient was confirmed only after the fifth nasopharyngeal swab sample to detect SARS-CoV-2 RNA. ${ }^{11}$ It can be seen that repeated swab testing may be useful for the diagnosis of initially negative SARS-CoV-2 RNA RT-PCR patients. If conditions permit, detection of multiple biological samples of suspected COVID-19 cases can improve the detection rate of SARS-CoV-2 RNA, which can guide clinical diagnosis and treatment.

\section{Conclusion}

Epidemiological investigation should continue when suspected COVID-19 patients are reported. If respiratory symptoms typical of COVID-19 are observed but the SARS-CoV-2 RNA test is negative, then reasonable isolation protection measures should be arranged immediately to prevent further spread of the virus.

\section{Ethical Approval}

The research related to human use has been complied with all the relevant national regulations, institutional policies and in accordance the tenets of the Helsinki Declaration, and has been approved by the medical ethics committee of the First Affiliated Hospital of University of South China.

\section{Informed Consent}

Written informed consent was obtained from the patient presented herein, for publication of this report and any accompanying images and patient details.

\section{Acknowledgments}

We wish to express our sincere thanks to Xiaoping Long for his help with language and constructive suggestions for this article.

\section{Author Contributions}

Xialing Huang and Jun Zi drafted the article; Jun Zi and Ping Liu collected and analyzed the data; Xiaoping Long conceived of and designed the study, acquired the funding, and revised the manuscript for important intellectual content; all authors read and approved the final version of the manuscript. All authors contributed to data analysis, drafting or revising the article, have agreed on the journal to which the article will be submitted, gave final approval of the version to be published, and agree to be accountable for all aspects of the work.

\section{Funding}

Supported by the Construction of an Innovative Province with a Special Emergency Response Topic in 2020, No. 2020SK3010, and the Fund Project of University of South China for Prevention and Control of COVID-19, No. 2020-27.

\section{Disclosure}

The authors report no conflicts of interest in this work. 


\section{References}

1. Contini C, Di Nuzzo M, Barp N, et al. The novel zoonotic COVID-19 pandemic: an expected global health concern. $J$ Infect Dev Ctries. 2020;14(3):254-264. doi:10.3855/jidc.12671

2. National Health Commission. Diagnosis and treatment protocol for novel coronavirus pneumonia (Trial Version 7). Chin Med J (Engl). 2020;133(9):1087-1095. doi:10.1097/CM9.00000000000 00819

3. Guan WJ, Ni ZY, Hu Y, et al. China Medical Treatment Expert Group for Covid-19. Clinical characteristics of coronavirus disease 2019 in China. $N$ Engl J Med. 2020;382(18):1708-1720. doi:10.1056/NEJM oa2002032

4. $\mathrm{Xu} \mathrm{X,} \mathrm{Yu} \mathrm{CC,} \mathrm{Qu} \mathrm{J,} \mathrm{et} \mathrm{al.} \mathrm{Imaging} \mathrm{and} \mathrm{clinical} \mathrm{features} \mathrm{of} \mathrm{patients}$ with 2019 novel coronavirus SARS-CoV-2. Eur J Nucl Med Mol Imaging. 2020;47(5):1275-1280. doi:10.1007/s00259-020-04735-9

5. Majumder J, Minko T. Recent developments on therapeutic and diagnostic approaches for COVID-19. AAPS J. 2021;23(1):14. doi:10.1208/s12248-020-00532-2
6. Li YF, Yao L, Li JW, et al. Stability issues of RT-PCR testing of SARS-CoV-2 for hospitalized patients clinically diagnosed with COVID-19. J Med Virol. 2020;92(7):903-908. doi:10.1002/jmv.25786

7. Wu J, Liu JS, Li SJ, et al. Detection and analysis of nucleic acid in various biological samples of COVID-19 patients. Travel Med Infect Dis. 2020;37:101673. doi:10.1016/j.tmaid.2020.101673

8. Torretta S, Zuccotti G, Cristofaro V, et al. Diagnosis of SARS-CoV-2 by RT-PCR using different sample sources: review of the literature. Ear Nose Throat J. 2021;100(2_suppl):131S-138S. doi:10.1177/ 0145561320953231

9. Zhang PY, Cai Z, Wu WB, et al. The novel coronavirus (COVID-19) pneumonia with negative detection of viral ribonucleic acid from nasopharyngeal swabs: a case report. BMC Infect Dis. 2020;20 (1):317. doi:10.1186/s12879-020-05045-z.

10. Xiao AT, Tong YX, Zhang S. False negative of RT-PCR and prolonged nucleic acid conversion in COVID-19: rather than recurrence. J Med Virol. 2020;92(10):1755-1756. doi:10.1002/jmv.25855

11. Chen LD, Li H, Ye YM, et al. A COVID-19 patient with multiple negative results for PCR assays outside Wuhan, China: a case report. BMC Infect Dis. 2020;20(1):517. doi:10.1186/s12879-020-05245-7
Infection and Drug Resistance

\section{Publish your work in this journal}

Infection and Drug Resistance is an international, peer-reviewed openaccess journal that focuses on the optimal treatment of infection (bacterial, fungal and viral) and the development and institution of preventive strategies to minimize the development and spread of resistance. The journal is specifically concerned with the epidemiology of

\section{Dovepress}

antibiotic resistance and the mechanisms of resistance development and diffusion in both hospitals and the community. The manuscript management system is completely online and includes a very quick and fair peerreview system, which is all easy to use. Visit http://www.dovepress.com/ testimonials.php to read real quotes from published authors. 